\title{
Modelo Dinámico Computacional de Metaestructura Piezoeléctrica Curva
}

\section{Computational Dynamic Model of Curved Piezoelectric Metastructure}

Presentación: 6-7/10/2020

\section{Doctorando:}

\section{Lucas Di Giorgio}

Centro de Investigación en Mecánica Teórica y Aplicada, Facultad Regional Bahía Blanca, Universidad Tecnológica Nacional - Argentina.

Idigiorgio@frbb.utn.edu.ar

\section{Director:}

\section{Marcelo Piovan}

\section{Co-Directora:}

\section{Marta Vidal}

\section{Resumen}

El objetivo del presente trabajo es el desarrollo de un modelo computacional unidimensional de una metaestructura curva de material piezoeléctrico en configuración bimorfo. El modelo será capaz de calcular la respuesta dinámica de la metaestructura en las 3 dimensiones del espacio, de manera precisa y con bajo costo computacional. El mismo deriva de la teoría de la elasticidad lineal y la piezoelectricidad general, y se obtiene por medio de la discretización en elementos finitos de una formulación débil, obtenida en base al Principio de Hamilton para sistemas electromecánicos.

Palabras Clave: Metaestructura, viga curva, piezoeléctrico, bandgap.

\begin{abstract}
The objective of the present work is the development of a one-dimensional computational model of a curved metastructure of piezoelectric material in a bimorph configuration. The model will be able to calculate the dynamic response of the metastructure in the 3 dimensions of the space, accurately and with low computational cost. It derives from the theory of linear elasticity and general piezoelectricity, and is obtained through finite element discretization of a weak formulation, obtained based on the Hamilton Principle for electromechanical systems.
\end{abstract}

Keywords: Metaestructure, curved beam, piezoelectric, bandgap.

\section{Introducción}

Las metaestructuras de resonadores internos han sido estudiadas durante años por su capacidad de generar bandas de atenuación para la propagación de vibraciones mecánicas, llamadas bandgaps. Una interesante manera de lograr el mismo efecto, sin necesidad de utilizar masas de gran tamaño, es replicar el resonador con amortiguadores piezoeléctricos [Lesieutre, 1998]. El enfoque típico para el estudio de metaestructuras es considerarlas de longitud infinita, permitiendo de esta manera la utilización del teorema de Bloch para obtener su estructura de bandas 
[Brillouin, 2003]. En la implementación, sin embargo, las dimensiones finitas exigen el estudio de la dinámica mediante de modelos que incorporen tanto a las dimensiones reales, así como también a las condiciones de borde [Erturk and Inman, 2011], [Surabhi, 2014].

A continuación se presenta un modelo computacional 1D en elementos finitos [Bathe, 1982] de una metaestructura curva de radio constante basada en el modelo analítico propuesto por Sugino [Sugino et al., 2017], agregando curvatura y la capacidad de reproducir desplazamientos en 3 dimensiones. La misma está constituida por un arreglo de bloques piezoeléctricos en configuración Bimorfo paralelo, realimentados cada uno con una inductancia eléctrica asociada, a fin de generar un circuito resonante LC con la capacidad interna de cada bloque, convirtiéndolos en bloques resonadores electromecánicos.

\section{Desarrollo del Modelo}

\section{Descripción}

La Fig.1 representa la metaestructura curva, constituida por piezoeléctricos en configuración bimorfo paralelo, a través de un material sustrato portante de sección rectangular. Los electrodos de cada piezoeléctrico están seccionados y aislados eléctricamente en segmentos de longitud $\Delta x$, formando bloques electromecánicos llamados bloques resonadores. Cada bloque posee su propia inductancia $\mathrm{L}_{k}$ conectada a los terminales del mismo. El modelo teórico se basa en las siguientes hipótesis:

- La curvatura de se ubica sobre el plano $x y$ y su radio de curvatura $R$ es constante.

- La sección trasversal es regular y rígida en su propio plano.

- Se consideran pequeños desplazamientos y elasticidad lineal.

- Se considera el esfuerzo cortante producido por flexión y torsión.

- Se define una función de alabeo referida al centroide.

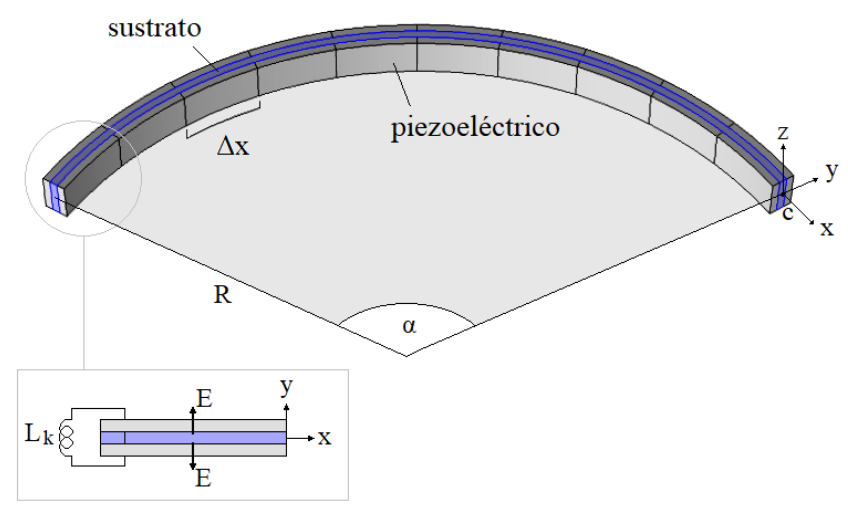

Figura 1: Esquema de la metaestructura curva.

De las hipótesis se desprende el siguiente campo de desplazamientos [Piovan and Cortinez, 2007]:

$$
\left\{\begin{array}{l}
u_{x} \\
u_{y} \\
u_{z}
\end{array}\right\}=\left\{\begin{array}{c}
u_{x c}-\omega \phi_{w} \\
u_{y c} \\
u_{z c}
\end{array}\right\}+\left[\begin{array}{ccc}
0 & -\phi_{z} & \phi_{y} \\
\phi_{z} & 0 & -\phi_{x} \\
-\phi_{y} & \phi_{x} & 0
\end{array}\right]\left\{\begin{array}{l}
0 \\
y \\
z
\end{array}\right\}
$$

donde

$$
\phi_{x}=\theta_{x}, \phi_{y}=\theta_{y}, \phi_{z}=\theta_{z}-\frac{u_{x c}}{R}, \phi_{w}=\theta_{w}+\frac{\theta_{y}}{R}
$$


siendo $\left\{u_{x c}, u_{y c}, u_{z c}\right\}$ los desplazamientos del centro de referencia, $\left\{\theta_{y}, \theta_{z}\right\}$ los parámetros de flexión, $\theta_{x}$ el ángulo de torsión y $\theta_{w}$ la intensidad de alabeo. La función de alabeo para una viga curva puede ser aproximada con la siguiente expresión [Yang and Kuo, 1987]:

$$
\omega=\bar{\omega} \mathcal{F} \quad \text { donde } \quad \mathcal{F}=\frac{R}{R+y}, \bar{\omega}=-x y
$$

donde $\bar{\omega}$ es la función de alabeo deducida del problema de torsión pura de Saint Venant [Piovan et al., 2012].

\section{Ecuaciones constitutivas y campo de deformaciones}

Considerando coincidencia entre los ejes cristalográficos y cartesianos, y teniendo en cuenta que $S_{y y}=S_{z z}=S_{y z}=0$, se obtienen las siguientes ecuaciones constitutivas reducidas para el piezoeléctrico [IEEE, 1988]:

$$
\left[\begin{array}{c}
T_{x x} \\
T_{x y} \\
T_{x z} \\
D_{y}
\end{array}\right]=\left[\begin{array}{cccc}
\bar{c}_{11}^{E} & 0 & 0 & -\bar{e}_{31} \\
0 & \bar{c}_{55}^{E} & 0 & 0 \\
0 & 0 & \bar{c}_{66}^{E} & 0 \\
\bar{e}_{31} & 0 & 0 & \bar{\varepsilon}_{33}^{S}
\end{array}\right]\left[\begin{array}{c}
S_{x x} \\
S_{x y} \\
S_{x z} \\
E_{y}
\end{array}\right]
$$

donde $\left\{\bar{c}_{11}^{E}, \bar{c}_{55}^{E}, \bar{c}_{66}^{E}\right\}\left[\mathrm{N} / \mathrm{m}^{2}\right]$ son las constantes de elasticidad longitudinal y trasversal evaluadas a campo eléctrico constante, $\bar{e}_{31}\left[\mathrm{Cb} / \mathrm{m}^{2}\right]$ la constante de acoplamiento, y $\bar{\varepsilon}_{33}^{S}[\mathrm{~F} / \mathrm{m}]$ la constante de permisividad eléctrica evaluada a deformación constante. La barra superior indica que la constante es reducida del modelo 3D hacia el 1D.

El campo de deformaciones de la estructura curva en función del campo de desplazamientos de la Ec.(1) se expresa como sigue [Piovan et al., 2012]:

$$
\left\{\begin{array}{c}
S_{x x} \\
S_{x y} \\
S_{x z}
\end{array}\right\}=\frac{1}{1+\frac{y}{R}}\left[\begin{array}{cccccccc}
1 & z & -y & -\omega & 0 & 0 & 0 & 0 \\
0 & 0 & 0 & 0 & 1 & 0 & \frac{\partial \bar{\omega}}{\partial y} & -\left(z+\frac{\partial \bar{\omega}}{\partial y}\right) \\
0 & 0 & 0 & 0 & 0 & 1 & \frac{\partial \bar{\omega}}{\partial z} & \left(y-\frac{\partial \bar{\omega}}{\partial z}\right)
\end{array}\right]\left\{\begin{array}{l}
\varepsilon_{D 1} \\
\varepsilon_{D 2} \\
\varepsilon_{D 3} \\
\varepsilon_{D 4} \\
\varepsilon_{D 5} \\
\varepsilon_{D 6} \\
\varepsilon_{D 7} \\
\varepsilon_{D 8}
\end{array}\right\}
$$

donde

$$
\begin{array}{llll}
\varepsilon_{D 1}=u_{x c}^{\prime}+\frac{u_{y c}}{R} & \varepsilon_{D 2}=\theta_{y}^{\prime}-\frac{\theta_{x}}{R} & \varepsilon_{D 3}=\theta_{z}^{\prime}-\frac{u_{x c}^{\prime}}{R} & \varepsilon_{D 4}=\theta_{w}^{\prime}+\frac{\theta_{y}^{\prime}}{R} \\
\varepsilon_{D 5}=u_{y c}^{\prime}-\theta_{z} & \varepsilon_{D 6}=u_{z c}^{\prime}+\theta_{y} & \varepsilon_{D 7}=\theta_{x}^{\prime}-\theta_{w} & \varepsilon_{D 8}=\theta_{x}^{\prime}+\frac{\theta_{y}}{R}
\end{array}
$$

\section{Principio de Hamilton}

El Principio de Hamilton para sistemas electromecánicos se expresa mediante el siguiente indicador variacional:

$$
\int_{t_{1}}^{t_{2}}\left[\delta\left(K^{*}+W_{e m}^{*}\right)+\delta W_{n c}\right] d t=0
$$

donde los términos del Lagrangiano $\left(K^{*}+W_{e m}^{*}\right)$ son:

$$
\begin{gathered}
K^{*}=\frac{1}{2} \int_{\Omega} \rho \dot{\boldsymbol{u}}^{T} \dot{\boldsymbol{u}} \frac{d \Omega}{\mathcal{F}} \\
W_{e m}^{*}=\frac{1}{2} \int_{\Omega}\left(\boldsymbol{E}^{T} \varepsilon \boldsymbol{E}+2 \boldsymbol{S}^{T} \boldsymbol{e} \boldsymbol{E}-\boldsymbol{S}^{T} \boldsymbol{c} \boldsymbol{S}\right) \frac{d \Omega}{\mathcal{F}}
\end{gathered}
$$


y el trabajo eléctrico no conservativo producido por las cargas eléctricas en los electrodos de cada bloque resonador es:

$$
W_{n c e}=\frac{1}{2} Q_{k} v_{k}
$$

donde $Q_{k}$ es la carga neta y $v_{k}$ el voltaje de cada bloque. Reemplazando Ec.(7), Ec.(8) y Ec.(9) en Ec.(6) se obtiene la formulación débil:

$$
\int_{\Omega}\left(\delta \boldsymbol{E}^{T} \varepsilon \boldsymbol{E}+\delta \boldsymbol{E}^{T} \boldsymbol{e}^{T} \boldsymbol{S}+\delta \boldsymbol{S}^{T} \boldsymbol{e} \boldsymbol{E}-\delta \boldsymbol{S}^{T} \boldsymbol{c} \boldsymbol{S}-\rho \delta \boldsymbol{u}^{T} \ddot{\boldsymbol{u}}\right) \frac{d \Omega}{\mathcal{F}}+Q_{k} \delta v_{k}=0
$$

\section{Discretización en elementos finitos}

La formulación en elementos finitos es obtenida mediante la discretización de la Ec.(10) considerando el campo eléctrico $E_{y}=v_{k} / h_{p z}$, donde $h_{p z}$ corresponden a la altura de la capa piezoeléctrica respectivamente. La discretización se realiza utilizando elementos isoparamétricos de 3 nodos y funciones de forma cuadráticas. El vector de las variables cinemáticas nodales del elemento puede ser expresado como:

$$
\bar{U}_{e}^{T}=\left\{\bar{U}_{e 1}^{T}, \bar{U}_{e 2}^{T}, \bar{U}_{e 3}^{T}\right\}, \quad \bar{U}_{e j}^{T}=\left\{u_{x c_{j}}, u_{y c_{j}}, \theta_{z_{j}}, u_{z c_{j}}, \theta_{y_{j}}, \theta_{x_{j}}, \theta_{w_{j}}\right\}, \quad j=1,2,3
$$

El método de elementos finitos deriva en las siguiente ecuación dinámica de estado estacionario de la metaestructura paralelo

$$
\left[\mathbf{K}-\omega^{2} \mathbf{M}+j \omega \mathbf{C}+\sum_{k=1}^{N_{b}} j \omega \mathbf{T}_{k}\left(j \omega \mathbf{C}+\frac{1}{j \omega \mathrm{L}_{k}}\right)^{-1}\right] \widehat{\boldsymbol{U}}=0
$$

donde $\widehat{\boldsymbol{U}}$ es el vector de amplitud de las variables cinemáticas en estado estacionario, $\mathbf{K}$ y $\mathbf{M}$ son las matrices de rigidez y elasticidad, $\mathbf{C}=\eta_{1} \mathbf{M}+\eta_{2} \mathbf{K}$ la matriz de amortiguamiento de [Rayleigh and Strutt, 1945], $\mathbf{T}_{k}$ la matriz de acoplamiento asociada a cada bloque resonador, y C y $\mathrm{L}_{k}$ los valores de capacidad e inductancia eléctrica asociados a cada bloque resonador. En el caso que $\mathrm{L}_{k}=\mathrm{L}$, la metaestructura se considera sintonizada, generando bandgap en la respuesta de propagación vibratoria, cuya frecuencia de sintonía se calcula como:

$$
\omega_{t}=\frac{1}{\sqrt{\mathrm{LC}}}
$$

\section{Validación del Modelo}

En esta sección se compara el el modelo numérico 1D con el modelo analítico propuesto por Sugino en [Sugino et al., 2017]. Para este estudio, ambas metaestructuras se componen de material piezoeléctrico PZT-5A: $C_{11}=61 G P a, C_{44}=C_{55}=21 G P a, \epsilon_{33}=13,3 e^{-9} \mathrm{~F} / \mathrm{m}$, $e_{31}=-12,3 \mathrm{C} / \mathrm{m}^{2}, \rho=7750 \mathrm{Kg} / \mathrm{m}^{3}$ y sustrato de aluminio: $E=70 G P a, G=26,32 G P a$, $\nu=0,33, \rho=2700 \mathrm{Kg} / \mathrm{m}^{3}$, cuyas dimensiones son $l=100 \mathrm{~mm}, b=10 \mathrm{~mm}, h_{p z}=0,3 \mathrm{~mm}, \mathrm{y}$ $h_{s}=0,1 \mathrm{~mm}$. La metaestructura se encuentra sintonizada a $\omega_{t}=100 \omega_{1}$. La Fig. 2 muestra los resultados computacionales de la propagación para diferente número de resonadores $N_{b}$, definiendo propagación como el cociente del desplazamiento de un punto del extremo libre $\mathrm{x}_{L}=(L, y, z)$ y el desplazamiento de un punto del extremo empotrado a la base $\mathrm{x}_{0}=(0,0,0)$, cuando la estructura es excitada con movimiento armónico desde la base, es decir

$$
T R(\omega)=\left|\frac{u\left(\mathrm{x}_{L}\right)}{u\left(\mathrm{x}_{0}\right)}\right|=\left|\frac{u_{L}}{u_{0}}\right|
$$

Claramente puede observarse la similitud entre las respuestas, la cual incluye la zona de bandgap, sin embargo debe tenerse en cuenta que el modelo analítico solo contempla movimientos en un 
solo plano para estructuras exclusivamente rectas, mientras que el presente modelo 1D se extiende para estructuras curvas y puede representar desplazamientos en 3 direcciones.

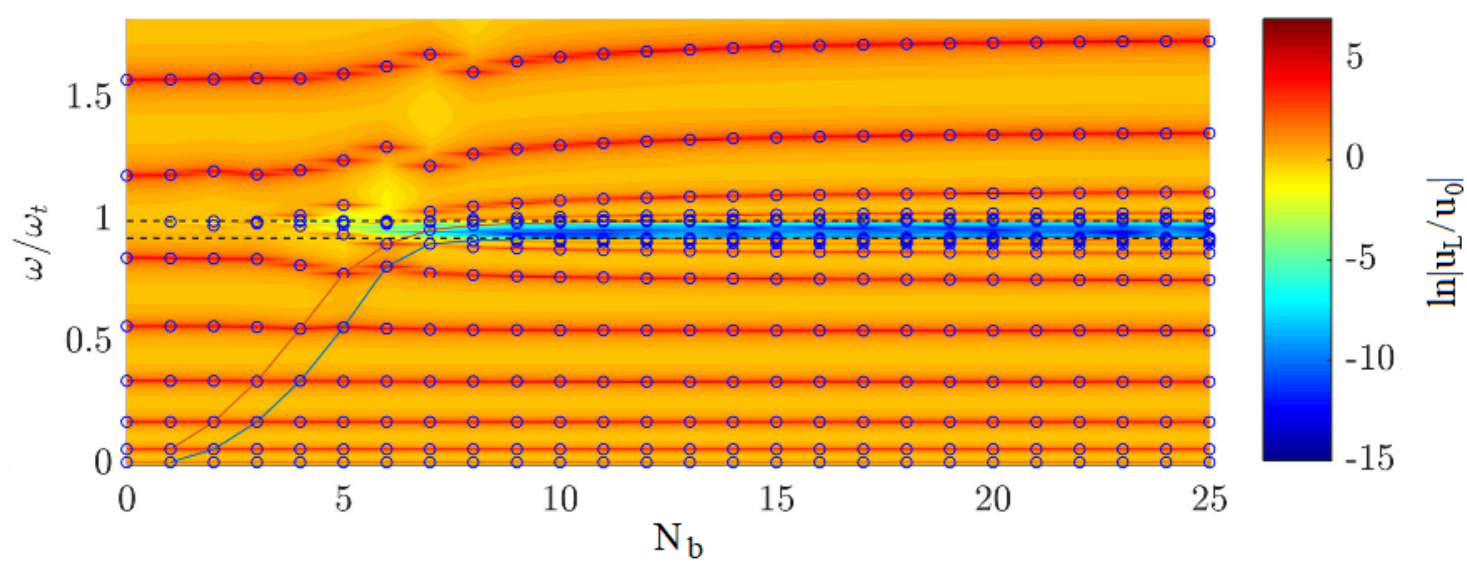

(a)

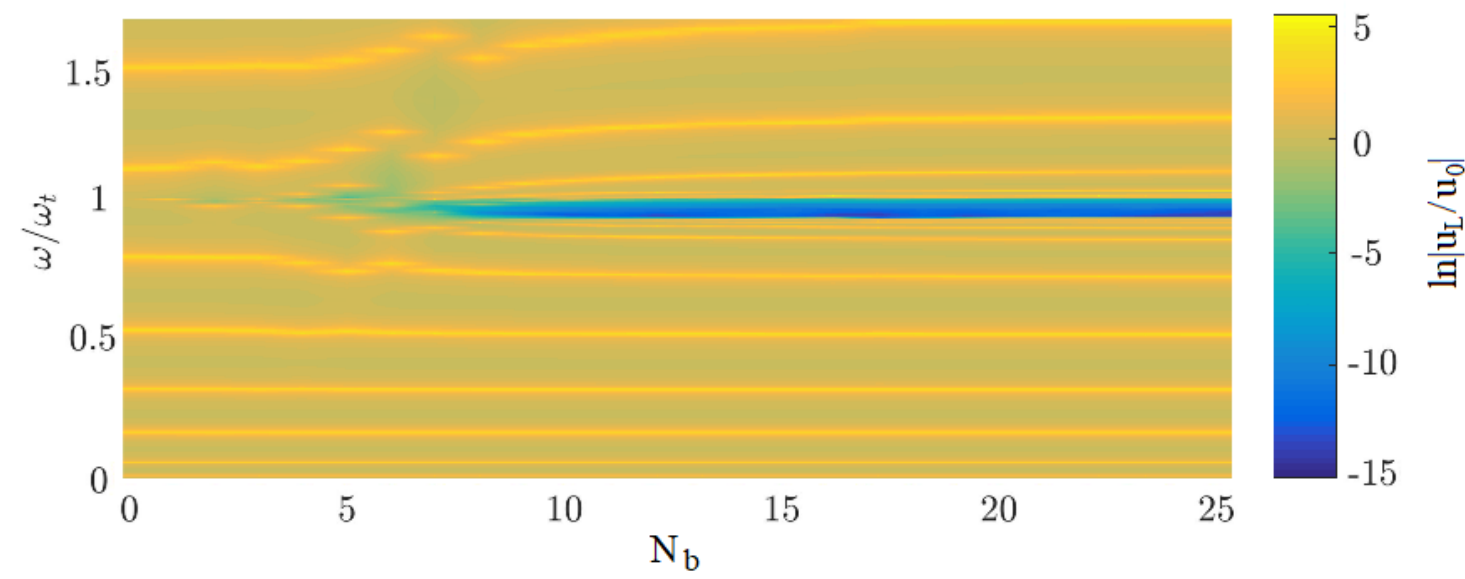

(b)

Figura 2: Propagación y frecuencias vs $N_{b}$. (a) Modelo analítico, (b) Modelo computacional.

\section{Conclusiones}

El presente trabajo se desarrolló un modelo de metaestructura piezoeléctrica formulado en elementos finitos, basado en el trabajo de Sugino, con la posibilidad de modelar curvatura en la misma, y con la habilidad de reproducir desplazamientos en 3 dimensiones. El modelo cuenta con las siguientes ventajas:

- Bajo costo computacional, permitiendo realizar análisis dinámicos con métodos recursivos, como es el caso del método de Monte Carlo para estudios de propagación de incertidumbre (aproximadamente 20seg. en una computadora de escritorio).

- Permite modelar con notable exactitud la dinámica de la metaestructura curvada. Esto es algo muy difícil de conseguir mediante programas de elementos finitos comerciales, debido a la necesidad de modelar electrostática y mecánica en simultaneo, manteniendo además la anisotropía del material piezoeléctrico en toda la curvatura.

- La curvatura de la metaestructura puede realizarse sobre el plano $x z$ mediante un simple cambio de variables en la integración de la sección de los elementos finitos. 


\section{Referencias}

[Bathe, 1982] Bathe, K. (1982). Finite Element procedures in Engineerign Analysis. PrenticeHall, Englewood Cliffs, New Jersey.

[Brillouin, 2003] Brillouin, L. (2003). Wave propagation in periodic structures: electric filters and crystal lattices. Courier Corporation.

[Erturk and Inman, 2011] Erturk, A. and Inman, D. J. (2011). Piezoelectric energy harvesting. John Wiley \& Sons.

[IEEE, 1988] IEEE (1988). An American National Standard: IEEE Standard on Piezoelectricity Standard. IEEE.

[Lesieutre, 1998] Lesieutre, G. A. (1998). Vibration damping and control using shunted piezoelectric materials. Shock and Vibration Digest, 30(3):187-195.

[Piovan and Cortinez, 2007] Piovan, M. and Cortinez, V. (2007). Mechanics of thin-walled curved beams made of composite materials, allowing for shear deformability. Thin-Walled Structures, 45:759-789.

[Piovan et al., 2012] Piovan, M. T., Domini, S., and Ramirez, J. M. (2012). In-plane and outof-plane dynamics and buckling of functionally graded circular curved beams. Composite Structures, 94(11):3194-3206. Cited By :26.

[Rayleigh and Strutt, 1945] Rayleigh, B. and Strutt, J. W. (1945). The theory of sound. Dover.

[Sugino et al., 2017] Sugino, C., Leadenham, S., Ruzzene, M., and Erturk, A. (2017). An investigation of electroelastic bandgap formation in locally resonant piezoelectric metastructures. Smart Materials and Structures, 26(5):055029.

[Surabhi, 2014] Surabhi, A. (2014). Finite element beam model for piezoelectric energy harvesting using higher order shear deformation theory. $\mathrm{PhD}$ thesis, Graduate School of Clemson University.

[Yang and Kuo, 1987] Yang, Y.-B. and Kuo, S.-R. (1987). Effect of curvature on stability of curved beams. Journal of structural Engineering, 113(6):1185-1202. 